\title{
Lung fluid transport in aquaporin-1 and aquaporin-4 knockout mice
}

\author{
Chunxue Bai, Norimasa Fukuda, Yualin Song, Tonghui Ma, Michael A. Matthay, \\ and A.S. Verkman
}

Departments of Medicine and Physiology, Cardiovascular Research Institute, University of California-San Francisco, San Francisco, California 94143-0521, USA

Address correspondence to: A.S. Verkman, Cardiovascular Research Institute, 1246 Health Sciences East Tower, Box 0521, University of California-San Francisco, San Francisco, California 94143-0521, USA. Phone: (415) 476-8530; Fax: (415) 665-3847;

E-mail: verkman@itsa.ucsf.edu; http://www.ucsf.edu/verklab

Received for publication May 29, 1998, and accepted in revised form December 22, 1998.

\begin{abstract}
The mammalian lung expresses water channel aquaporin-1 (AQP1) in microvascular endothelia and aquaporin-4 (AQP4) in airway epithelia. To test whether these water channels facilitate fluid movement between airspace, interstitial, and capillary compartments, we measured passive and active fluid transport in AQP1 and AQP4 knockout mice. Airspace-capillary osmotic water permeability $\left(\mathrm{P}_{\mathrm{f}}\right)$ was measured in isolated perfused lungs by a pleural surface fluorescence method. $\mathrm{P}_{\mathrm{f}}$ was remarkably reduced in AQP1 (-/-) mice (measured in $\mathrm{cm} / \mathrm{s} \times 0.001$, SE, $n=5-10: 17 \pm 2[+/+] ; 6.6 \pm 0.6 \mathrm{AQP} 1[+/-] ; 1.7 \pm 0.3 \mathrm{AQP} 1[-/-] ; 12 \pm 1 \mathrm{AQP} 4$ $[-/-])$. Microvascular endothelial water permeability, measured by a related pleural surface fluorescence method in which the airspace was filled with inert perfluorocarbon, was reduced more than 10-fold in AQP1 (-/-) vs. (+/+) mice. Hydrostatically induced lung interstitial and alveolar edema was measured by a gravimetric method and by direct measurement of extravascular lung water. Both approaches indicated a more than twofold reduction in lung water accumulation in AQP1 (-/-) vs. (+/+) mice in response to a 5- to $10-\mathrm{cm} \mathrm{H}_{2} \mathrm{O}$ increase in pulmonary artery pressure for five minutes. Active, near-isosmolar alveolar fluid absorption $\left(\mathrm{J}_{\mathrm{v}}\right)$ was measured in in situ perfused lungs using ${ }^{125} \mathrm{I}$-albumin as an airspace fluid volume marker. $\mathrm{J}_{\mathrm{v}}$ (measured in percent fluid uptake at $\left.30 \mathrm{~min}, n=5\right)$ in $(+/+)$ mice was $6.0 \pm 0.6\left(37^{\circ} \mathrm{C}\right)$, increased to $16 \pm 1$ by $\beta$-agonists, and inhibited to less than 2.0 by amiloride, ouabain, or cooling to $23^{\circ} \mathrm{C}$. $\mathrm{J}_{\mathrm{v}}$ (with isoproterenol) was not affected by aquaporin deletion (18.9 $\pm 2.2[+/+] ; 16.4 \pm 1.5 \mathrm{AQP} 1[-/-] ; 16.3 \pm 1.7$ AQP4 [-/-]). These results indicate that osmotically driven water transport across microvessels in adult lung occurs by a transcellular route through AQP1 water channels and that the microvascular endothelium is a significant barrier for airspace-capillary osmotic water transport. AQP1 facilitates hydrostatically driven lung edema but is not required for active near-isosmolar absorption of alveolar fluid.
\end{abstract}

J. Clin. Invest. 103:555-561 (1999).

\section{Introduction}

Substantial quantities of fluid move across epithelial and endothelial barriers in lung. In the perinatal lung, fluid absorption from the airspaces occurs in preparation for alveolar respiration (1). In the adult lung, movement of salt and water between the airspace and capillary compartments is required for control of airspace hydration. The formation and resolution of clinical pulmonary edema involve fluid movements among the airspace, interstitial, and capillary compartments (2-4). Considerable progress has been made in understanding the molecular mechanisms of salt movement between the airspace and capillaries. Recent work has begun to define the role of the $\mathrm{ENaC}$ sodium channel (5), the $\mathrm{ClC}-2$ and CFTR $\mathrm{Cl}^{-}$channels $(6,7)$, and the $\mathrm{Na}^{+} / \mathrm{K}^{+}$pump $(8,9)$.

There have been recent advances in understanding the molecular mechanisms of water movement in lung. A family of (currently) 10 related molecular water channels called aquaporins has been identified in mammals (reviewed in refs. 10-12). The aquaporins are small hydrophobic membrane proteins $\left(M_{r} \sim 30,000\right)$ with homology to the major intrinsic protein of lens fiber. Three aquaporins have been localized in lung: AQP1 in microvascular endothelia and some pneumocytes (13-15), AQP4 in the basolateral membrane of airway epithelium (16), and AQP5 in the apical membrane of type I alveolar epithelial cells (17). Aquaporin-type water channels have not yet been identified at the basolateral surface of alveolar epithelium or at the apical membrane of airway epithelia. The specific localization of aquaporins to endothelial and epithelial cells suggests a role in water movement between airspace, interstitial, and capillary compartments. Other indirect evidence supporting a physiological role for aquaporins in lung includes the increase in aquaporin expression (18-20) and lung water permeability (21) around the time of birth, and the high water permeability of alveolar $(13,22)$, microvascular (23), and airway (24) barriers. Recently, immunopurified type I alveolar epithelial cells were found to have an exceptionally high water permeability, with biophysical properties indicative of molecular water channels (25).

We have developed quantitative pleural surface fluorescence methods to measure osmotic water permeability of the airspace-capillary barrier (22) and microvascular endothelia (23) in intact lungs of small animals. To measure osmotically driven water movement between the air- 
space and capillary compartments, the airspace is filled with aqueous fluid containing a membrane impermeant fluorescent indicator. In response to a change in pulmonary artery perfusate osmolality, water moves into or out of the airspaces, producing a change in indicator concentration (proportional to airspace fluid osmolality). Airspace-capillary osmotic water permeability $\left(\mathrm{P}_{\mathrm{f}}\right)$ in rat and mouse lung was found to be high $(\sim 0.02 \mathrm{~cm} / \mathrm{s})$, weakly temperature-dependent, and mercurial-inhibitable (22). To measure the water permeability of lung microvessels, the airspace is filled with an inert perfluorocarbon, and the pulmonary artery is perfused with solutions of different osmolalities containing fluorescent indicators (23). Microvascular osmotic water permeability is determined from the prompt change in fluorescence in response to a change in perfusate osmolality. Using a mathematical model to relate the fluorescence change to water permeability, microvascular $\mathrm{P}_{\mathrm{f}}$ in mouse lung was found to be high $(\sim 0.03 \mathrm{~cm} / \mathrm{s})$ and inhibited by mercurials. Although these measurements provided quantitative values for water permeabilities, they give no information about the relative contributions of transcellular vs. paracellular water transport across lung microvessels, nor do they define the relative barrier properties of epithelial vs. endothelial cell layers or the contribution of specific aquaporins.

The primary goal of this study was to test the hypothesis that aquaporin-type water channels facilitate osmotically driven and hydrostatically driven fluid transport between the airspace, interstitial, and capillary compartments. The specific issue of whether water moves across lung microvessels by a transcellular route involving AQP1 was also addressed, as well as whether the microvascular endothelium poses a significant barrier to airspace-capillary water transport. The potential contribution of AQP1 and AQP4 in active, near-isosmolar alveolar fluid absorption was also examined. Water permeability measurements were done using the methods already mentioned here on transgenic knockout mice deficient in AQP1 and AQP4. In addition, methods were adapted to quantify hydrostatically induced lung edema and active isosmolar alveolar fluid absorption. We found that AQP1 provides a quantitatively important route for osmotically and hydrostatically driven water movement between the airspace, interstitial, and capillary compartments, but that AQP1 is not required for active, isosmolar alveolar fluid absorption.

\section{Methods}

Transgenic mice. Transgenic knockout mice deficient in AQP1 and AQP4 proteins were generated by targeted gene disruption as described previously $(26,27)$. The AQP1 knockout mice were reported to be mildly growth retarded compared with wild-type mice, and they manifest a severe defect in urinary concentrating ability when deprived of water (27). Analysis of kidney proximal tubule function in vitro and in vivo indicated fivefold-reduced transepithelial osmotic water permeability and defective active, near-isosmolar fluid absorption (28). The AQP4 knockout mice appear to have a grossly normal phenotype except for a mild urinary concentrating defect (26) that is related to decreased transepithelial water permeability in inner medullary collecting duct (29).

Measurements were done in litter-matched mice (8-10 weeks of age, 20-25 g body weight) produced by intercrossing of CD1 heterozygotes. Genotype analysis of tail DNA was done by PCR at 5 days of age. The investigators were blinded to genotype information for all comparative transport measurements.

Isolated lung perfusion. Mice were euthanized with intraperitoneal pentobarbital $(150 \mathrm{mg} / \mathrm{kg})$. The trachea was cannulated with polyethylene PE-50 tubing, and the pulmonary artery with PE-90 tubing. Tubings were secured with suture to prevent fluid leakage. The left atrium was transected. The heart and lungs were moved en bloc to a perfusion chamber for observation by epifluorescence microscopy. The pulmonary artery was gravity-perfused at constant pressure $\left(25-35 \mathrm{~cm} \mathrm{H}_{2} \mathrm{O}\right)$ at room temperature. More than $80 \%$ of lung perfusions were successful; generally, the unsuccessful perfusions resulted from improper catheter insertion resulting in obvious fluid leak or poor perfusion. For airspace-capillary water permeability measurement, the airspace was filled with $0.5 \mathrm{ml}$ HEPES-buffered Ringer's solution (HBR) (137 mM NaCl, $2.68 \mathrm{mM} \mathrm{KCl}, 1.25$ $\mathrm{mM} \mathrm{MgSO}_{4}, 1.82 \mathrm{mM} \mathrm{CaCl}_{2}, 5.5 \mathrm{mM}$ glucose, $12 \mathrm{mM}$ HEPES, $5 \%$ BSA; pH 7.4, $300 \mathrm{mOsm})$ containing FITC-dextran (70 kDa, $0.5 \mathrm{mg} / \mathrm{ml})(22)$. For microvascular endothelial water permeability measurement, the airspace was filled with $0.5 \mathrm{ml}$ of Fluorinert (3M, St. Paul, Minnesota, USA), an inert, water-immiscible fluorocarbon (23). The fluorescence intensity from a 3- to 5 -mm-diameter spot on the lung pleural surface was monitored with an inverted epifluorescence microscope using a fluorescein filter set. Signals were detected by a photomultiplier and then amplified, digitized, and recorded at a rate of $1 \mathrm{~Hz}$.

Osmotic water permeability measurements. For measurement of airspace-capillary osmotic water permeability, an airspace-perfusate osmotic gradient was generated by switching the perfusate between HBR and HBR containing $300 \mathrm{mM}$ sucrose. The time course of pleural surface fluorescence was monitored continuously. $\mathrm{P}_{\mathrm{f}}$ was computed from the relation $\mathrm{P}_{\mathrm{f}}=\left[\mathrm{d}\left(\mathrm{F} / \mathrm{F}_{\mathrm{o}}\right) / \mathrm{dt}\right]_{\mathrm{t}=0} /$ $\left[\left(S / V_{o}\right) v_{w} \Delta C\right]$, where $P_{f}(\mathrm{~cm} / \mathrm{s})$ is the osmotic water permeability
Figure 1

Airspace-capillary water permeability measured by a pleural surface fluorescence method. Isolated lungs were perfused continuously, and the airspace was filled with an isosmolar solution containing FITC-dextran (see Methods). (a) Representative time course data shown for lungs of mice of indicated genotypes. ( $\boldsymbol{b}$ ) Individual and averaged (mean $\pm \mathrm{SE}$ ) reciprocal half-time $\left(1 / t_{1 / 2}\right)$ for the fluorescence signal change with corresponding airspace-capillary osmotic water permeability coefficients $\left(P_{f}\right)$. The investigator was blinded to genotype for all transport measurements. Each point is the averaged data for two to six sets of measurements on an individual mouse. ${ }^{*} P<0.005$ vs. $(+/+)$; ${ }^{*} P<0.0001$ vs. $(+/+)$. a

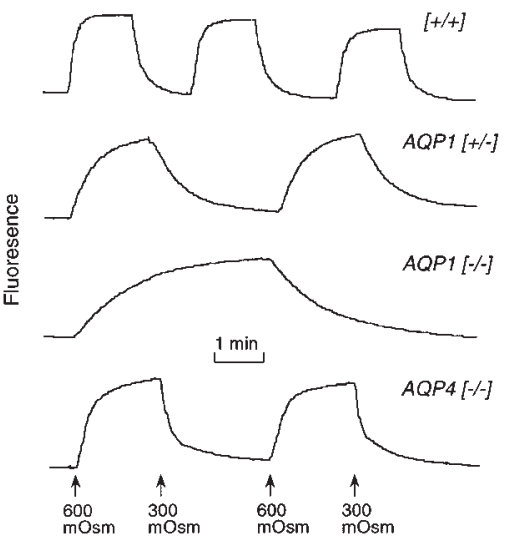

$\boldsymbol{b}$

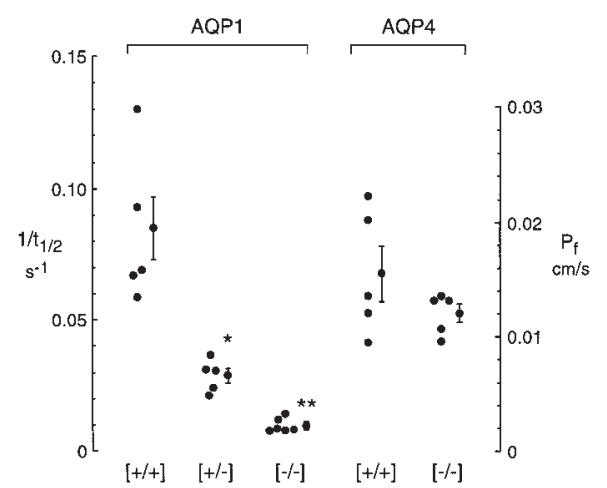


coefficient, $\left[\mathrm{d}\left(\mathrm{F} / \mathrm{F}_{\mathrm{o}}\right) / \mathrm{dt}\right]_{\mathrm{t}=0}$ is the initial slope of the relative fluorescence vs. time data, $S\left(\mathrm{~cm}^{2}\right)$ is surface area, $v_{w}\left(18 \mathrm{~cm}^{3} / \mathrm{mol}\right)$ is the partial molar volume of water, and $\Delta \mathrm{C}$ is the difference in osmolality between perfusate and airspace fluids (22).

Water transport across lung microvessels was measured by perfusing lungs with fluorescent solutions of specified osmolalities (23). Solutions consisted of HBR (300 mOsm) containing FITC-dextran $(0.5 \mathrm{mg} / \mathrm{ml}, 500 \mathrm{kDa}$; Sigma Chemical Co., St. Louis, Missouri, USA) and 0 or $200 \mathrm{mM}$ raffinose. Fluorescence intensities were identical in the isosmolar and hyperosmolar HBR solutions. Osmotic water movement was measured from the time course of fluorescence in response to switching between the isosmolar and hyperosmolar perfusates. Microvascular $P_{f}$ was estimated using a mathematical model relating $P_{f}$ to the fluorescence deflection, perfusate flow rate, osmotic gradient size, and lung geometry (Fig. 4 of ref. 23).

Measurement of bydrostatically driven lung edema. A gravimetric method was used to monitor water content continuously in the isolated perfused lung $(30,31)$. Lungs were suspended from a weight transducer (sensitivity less than $1 \mathrm{mg}$ ) and perfused as described earlier here. Fluid exiting the transected left atrium was drawn away continuously by a wax paper wick without affecting the weight measurement. The cumulative weight of fluid exiting the lung was recorded continuously using a weight transducer to determine perfusion rate. The pulmonary artery was gravity-perfused with HBR, and the trachea was transected and left open to air. The time course of lung weight was determined in response to an increase in pulmonary artery perfusate pressure from 8 to $18 \mathrm{~cm} \mathrm{H}_{2} \mathrm{O}$, established by setting reservoir height. Lung weight and perfusate flow were recorded using the gravimetric transducers and an analog-digital computer interface (Biopak Inc., Milwaukee, Wisconsin, USA). Lungs were weighed at the end of each experiment.

Lung edema was measured in an independent set of experiments by direct determination of extravascular lung water at a fixed time point. Lungs were perfused as already described here with HBR containing ${ }^{131} \mathrm{I}$-albumin as an extravascular fluid marker. Lungs were subject to a 10 -min perfusion at $5 \mathrm{~cm} \mathrm{H}_{2} \mathrm{O}$ pressure and then a 5-min perfusion at 5, 9, or $15 \mathrm{~cm} \mathrm{H}_{2} \mathrm{O}$. After perfusion, perfusate and lung radioactivity were measured to determine intravascular water content, and wet and dry lung and perfusate weights were measured. Extravascular lung water content was determined from (wet lung weight - pulmonary vessel content) / (dry lung weight - dried pulmonary vessel content), where pulmonary vessel content was determined from lung ${ }^{131} \mathrm{I}$ radioactivity/perfusate radioactivity, and dried pulmonary vessel content was determined from pulmonary vessel content multiplied by the ratio of dry/wet perfusate weight.

Measurement of active, near-isosmolar fluid absorption. Mice were euthanized as described earlier and maintained at $37 \pm 0.5^{\circ} \mathrm{C}$ using a heating pad, lamp, and monitoring thermistor. The left atrium was transected in situ, and the pulmonary artery was cannulated with PE-20 tubing. The trachea was cannulated with PE50 tubing through a tracheostomy. The pulmonary artery was gravity-perfused at $0.1-0.2 \mathrm{ml} / \mathrm{min}$ with $\mathrm{O}_{2}$-bubbled $\mathrm{HBR}$ at constant perfusion pressure of $8-10 \mathrm{~cm} \mathrm{H}_{2} \mathrm{O}$. The lungs were infused via the trachea with $0.5 \mathrm{ml}$ of HBR containing $1 \mu \mathrm{Ci}^{125} \mathrm{I}$-albumin, which was kept in contact with $100 \% \mathrm{O}_{2}$ at a constant pressure of 3-5 $\mathrm{cm} \mathrm{H}_{2} \mathrm{O}$. Fluid samples (5-200 $\left.\mu \mathrm{l}\right)$ were withdrawn at 2 and $30 \mathrm{~min}$ after the airspace infusion. In some experiments, isoproterenol $(0.1 \mathrm{mM})$, terbutaline $(10 \mathrm{mM})$, amiloride $(1 \mathrm{mM})$, or ouabain $(0.1 \mathrm{mM})$ was added to the perfusate and instillate solutions, or the experiment was done at $23^{\circ} \mathrm{C}$ instead of $37^{\circ} \mathrm{C}$. The percentage fluid absorption was computed from the ratio of sample radioactivities (measured in duplicate) at $30 \mathrm{vs.} 2 \mathrm{~min}$ after instillation, as was done previously (32).

Immunocytochemistry and immunoblot analysis. AQP1 and AQP4 localization in lung was done by immunoperoxidase using spe- cific polyclonal antibodies as described previously (16). Quantitative immunoblot analysis on lung homogenates was done as described $(26,27)$, using densitometry to compare integrated band intensities from lungs of (+/-) mice with those of serially diluted samples from $(+/+)$ mice.

\section{Results}

Measurements of lung water transport were done on wild-type mice $(+/+)$, AQP1 heterozygous mice $(+/-)$, AQP1 knockout (-/-) mice, and AQP4 knockout (-/-) mice. Immunostaining confirmed AQP1 localization mainly in capillary endothelia in (+/+) and (+/-) mice, as found previously (13-15), with no AQP1 in lungs from AQP1 (-/-) mice (not shown). AQP4 was localized to the basolateral membrane of airway epithelia in $(+/+)$ mice as reported previously (16), with no AQP4 in lungs from AQP4 (-/-) mice. Quantitative immunoblot analysis showed $30 \%-45 \%$ decreased AQP1 expression in lung homogenates from AQP1 (+/-) vs. (+/+) mice.

Measurements of osmotically driven water transport between the airspace and capillary compartments were done on isolated perfused lung by the pleural surface fluorescence method. The airspace was filled with an isosmolar solution containing fluorescein isothiocyanate (FITC)-dextran, and pleural surface fluorescence was monitored in response to changes in pulmonary artery perfusate osmolality. Fig. 1a shows representative time course data. In $(+/+)$ mice, there was a prompt increase in fluorescence $\left(t_{1 / 2} \sim 12\right.$ seconds) upon increase in perfusate osmolality from 300 to $600 \mathrm{mOsm}$ as water moved out of the fluid-filled airspace and concentrated the FITC-dextran. Fluorescence decreased promptly upon return of perfusate osmolality to $300 \mathrm{mOsm}$. The rates of fluorescence change were somewhat slowed in AQP1 $(+/-)$ mice $\left(t_{1 / 2}\right.$ $\sim 36$ seconds) and remarkably slowed in the AQP1 (-/-) mice $\left(t_{1 / 2} \sim 75\right.$ seconds). Fluorescence changes were prompt in AQP4 (-/-) mice. Differences in transport rates were not due to differences in alveolar size (diameter $40 \pm$ $5 \mu \mathrm{m}[+/+] ; 38 \pm 4 \mu \mathrm{m}$ AQP1 [-/-]), determined by pleural surface confocal microscopy as described previously (22).

Figure $1 b$ provides individual and averaged data from a series of mice in which the investigator was blinded to genotype information. Averaged $\mathrm{P}_{\mathrm{f}}$ values were (measured in $\mathrm{cm} / \mathrm{s} \times 0.001, \mathrm{SE}): 17 \pm 2(+/+) ; 6.6 \pm 0.6 \mathrm{AQP} 1(+/-)$; and $1.7 \pm 0.3 \mathrm{AQP} 1(-/-) . \mathrm{P}_{\mathrm{f}}$ in AQP4 (-/-) mice $(12 \pm 1$ $\mathrm{cm} / \mathrm{s})$ did not differ significantly from that in $(+/+)$ mice.

Because AQP1 is expressed primarily in alveolar microvessels, these results suggest that the decreased airspace-capillary $\mathrm{P}_{\mathrm{f}}$ in AQP1 (-/-) mice is related to a greatly reduced microvascular water permeability. This possibility was tested using a semiquantitative method to measure microvascular water permeability ( $\left.\mathrm{P}_{\mathrm{f}} \mathrm{ap}\right)$ in the intact lung. As described in Methods, the airspace of an isolated perfused lung was filled with an inert perfluorocarbon, and the pulmonary artery was perfused with solutions of different osmolalities containing FITC-dextran. Figure 2 shows that in response to an increase in perfusate osmolality from 300 to 500 mOsm, pleural surface fluorescence decreased promptly as the vascular compartment was diluted with fluid from the interstitial and intracellular spaces; the fluorescence signal then returned slowly, as no more water could be extracted. 


\section{Figure 2}

Microvascular water permeability in (+/+) and AQP1 (-/-) mice. The airspace of a perfused lung was filled with an inert perfluorocarbon, and the pulmonary artery was perfused with solutions of indicated osmolalities containing FITC-dextran (see Methods). Representative time course data for lungs of $(+/+)$ mice (left) and AQP1 (-/-) mice (right). Relative fluorescence changes $(\Delta F / F)$, which are related to microvascular osmotic water permeability, are summarized in the text.

Upon return of perfusate osmolality to $300 \mathrm{mOsm}$, the fluorescence signal increased promptly and then returned to baseline. As modeled previously (23), the relative amplitude of the prompt change in fluorescence signal $(\Delta \mathrm{F} / \mathrm{F})$ is related to the microvascular osmotic water permeability $\left(\mathrm{P}_{\mathrm{f}}\right.$ cap). The averaged signal amplitudes were greatly reduced in lungs from AQP1 (-/-) vs. $(+/+)$ mice $(\Delta \mathrm{F} / \mathrm{F}=0.12 \pm 0.02[+/+], 0.022 \pm 0.005[-/-]$; $n=4)$. From the previous mathematical modeling showing a nonlinear $\Delta \mathrm{F} / \mathrm{F}$ vs. water permeability relation (23), it is concluded that microvascular water permeability is reduced more than 10 -fold in AQP1 (-/-) vs. (+/+) mice. However, because the mathematical modeling required some assumptions about the surface area and uniformity of alveolar microvessels, quantitative comparison of absolute microvascular $\mathrm{P}_{\mathrm{f}}$ is probably not justified.

Several models of lung edema were used to determine whether aquaporin deletion affects the accumulation and removal of lung water. First, hydrostatic lung edema was studied from the time course of lung weight in response to changes in pulmonary artery pressure. Fig. $3 a$ shows the experimental setup. Lung weight was monitored continuously by a weight transducer, and the pulmonary artery was gravity-perfused with an isosmolar solution at specified hydrostatic pressure. As increase in hydrostatic pressure (from 8 to $18 \mathrm{~cm} \mathrm{H}_{2} \mathrm{O}$ ) was used to produce an increase in filtration and interstitial edema with minimal alveolar edema. Figure $3 b$ shows original weight curves from $(+/+)$ and AQP1 (-/-) mice. Lung weight was very
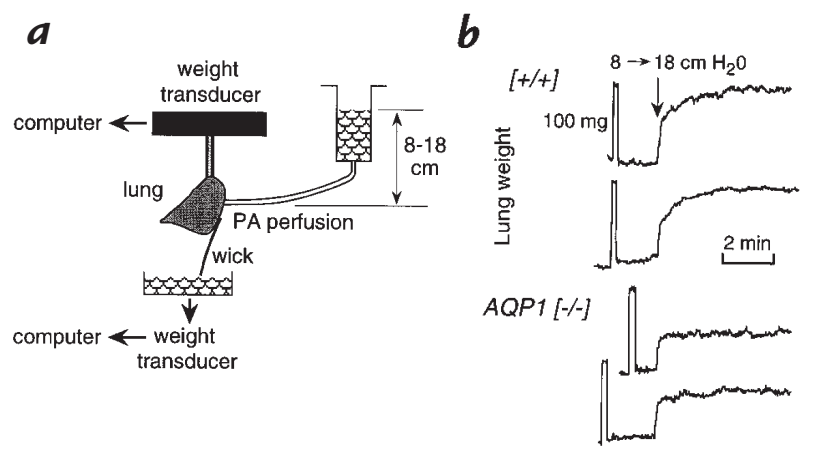

Figure 3

Gravimetric measurement of hydrostatically driven lung edema. (a) Lung weight was monitored continuously in isolated lungs perfused with an isosmolar solution at hydrostatic pressures of 8 or $18 \mathrm{~cm} \mathrm{H} \mathrm{H}_{2} \mathrm{O}$ as set by adjusting reservoir height (see Methods). (b) Original records of the time course of lung weight increase in response to change in pulmonary artery pressure from 8 to $18 \mathrm{~cm} \mathrm{H}_{2} \mathrm{O}$. Weight was calibrated in every experiment by briefly suspending a 100-mg weight standard from the lung.

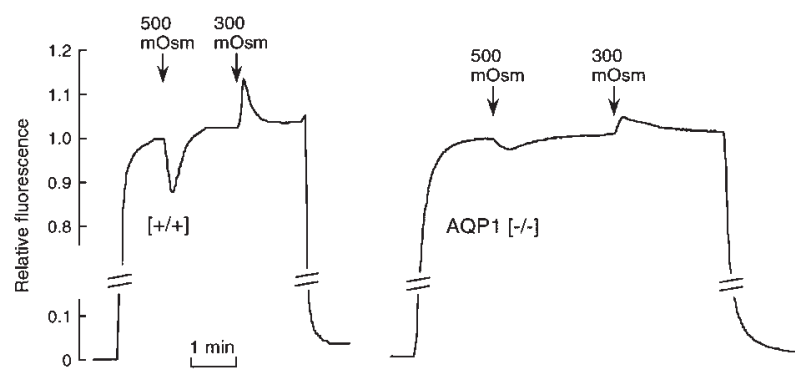

stable during the initial perfusion at a pressure of $8 \mathrm{~cm}$ $\mathrm{H}_{2} \mathrm{O}$. An internal calibration was done in each experiment by suspending a 100-mg weight standard from the lung. In response to the rapid increase in hydrostatic pressure, lung weight promptly increased in a biphasic manner. The initial rapid phase, which was complete in 10-15 seconds, represents primarily vascular recruitment/engorgement. The subsequent slower phase represents primarily accumulation of lung fluid. There was a consistently lower rate of lung fluid accumulation in the AQP1 (-/-) mice. The averaged lung weight increase between $15 \mathrm{sec}-$ onds and 3 minutes after the change in hydrostatic pressures was $45.6 \pm 4.2 \mathrm{mg}([+/+] ; \mathrm{SE}, n=8)$ and $14.5 \pm 2.4 \mathrm{mg}$ (AQP1 $[-/-] ; n=5)(P<0.001)$.

The increase in lung water in response to increased hydrostatic pressure was measured by an independent method that did not rely on the ability to resolve the vascular engorgement and edema phases. Extravascular lung water was measured directly from wet/dry weight ratios with correction for intravascular lung water using an ${ }^{131} \mathrm{I}-$ albumin marker (see Methods). Lungs were perfused at 5 $\mathrm{cm} \mathrm{H}_{2} \mathrm{O}$ pulmonary artery pressure for 10 minutes and then for 5 minutes at 5,9 , or $15 \mathrm{~cm} \mathrm{H}_{2} \mathrm{O}$ pressure. Figure 4 shows the lung extravascular wet/dry weight ratios. Whereas AQP1 deletion did not affect wet/dry weights in the absence of an increase in hydrostatic pressure, there was a significant reduction in lung water accumulation when perfusate pressure was raised to 9 or $15 \mathrm{~cm} \mathrm{H}_{2} \mathrm{O}$. Together with the gravimetric experiments discussed earlier here, these results implicate a role for AQP1 in hydrostatically induced fluid movement in lung.

To determine whether AQP1 or AQP4 deletion affects active isosmolar fluid reabsorption from the alveolar compartment, an in situ perfused lung method was developed to measure alveolar fluid absorption. The airspace was infused via the trachea with $0.5 \mathrm{ml}$ of an isosmolar solution containing ${ }^{125} \mathrm{I}$-albumin as a volume marker. The pulmonary artery was perfused with an isosmolar solution at constant pressure. Figure 5 (left) shows the data on individual $(+/+)$ mice for several protocols. Control absorption rate $J_{\mathrm{v}}$ (percent absorption at 30 minutes) was $6.0 \pm 0.9$, in general agreement with values reported in different mammals (reviewed in ref. 3). $\mathrm{J}_{\mathrm{v}}$ was increased significantly $(P<$ $0.01)$ by the $\beta$-agonists isoproterenol $(15.3 \pm 1.3)$ and terbutaline (15.6 \pm 1.1$)$. Absorption was inhibited strongly by the $\mathrm{Na}^{+}$channel inhibitor amiloride $(2.1 \pm 0.5)$, the $\mathrm{Na} / \mathrm{K}$ pump inhibitor ouabain $(0.1 \pm 1.2)$, and by carrying out the experiment (in the absence of inhibitors) at $23^{\circ} \mathrm{C}$ instead of $37^{\circ} \mathrm{C}(-0.6 \pm 0.6)$. These results establish the utility of the in situ perfusion method for measurement of active alveolar fluid absorption in mice. 
Similar measurements were done on a series of $(+/+)$, AQP1 (-/-), and AQP4 (-/-) mice in the presence of isoproterenol to maximize fluid absorption. As in the previous studies, the investigator was blinded to information about mouse genotype until the completion of all experiments. Fig. 5 (right) shows no significant effect of AQP1 or AQP4 deletion on the rate of active alveolar fluid absorption. $\mathrm{J}_{\mathrm{v}}$ values (percent absorption at 30 minutes) were as follows: $18.9 \pm 2.2(+/+) ; 16.4 \pm 1.5$ AQP1 (-/-); and $16.3 \pm 1.7$ AQP4 (-/-).

\section{Discussion}

As explained in the Introduction, several lines of indirect evidence suggest, but do not prove, that aquaporins play a role in lung physiology: aquaporins are expressed in lung epithelia and endothelia (13-17); water permeability is high in epithelia and endothelia where aquaporins are expressed $(13,22-24)$; and aquaporin expression (18-20) and function (21) increase near the time of birth. Analysis of the phenotype of aquaporin knockout mice enables direct examination of the functional role of aquaporins in lung. Such information cannot come from experiments using water transport inhibitors because suitable inhibitors for in vivo studies do not exist. Although water transport through AQP1 and AQP5 is inhibited by $\mathrm{HgCl}_{2}$ and related organic mercurials, these compounds are too toxic and nonspecific for use in living animals or intact organs. Inhibitors have not been identified for AQP4, whose water-transporting function is not inhibited by mercurials because a critical cysteine residue present in AQP1 and AQP5 is absent in AQP4 (33). The AQP1 and AQP4 knockout mice studied here have already provided a substantial body of information about the urinary concentrating mechanism in kidney (26-29).

The osmotic water permeability of lung microvessels was decreased by more than 10-fold in AQP1 knockout mice compared with wild-type mice. Airspace-capillary $\mathrm{P}_{\mathrm{f}}$, which reflects the composite microvascular, interstitial, and epithelial barriers, was decreased by $\sim 10$-fold in the AQP1 knockout mice. There was little effect of deletion of the airway water channel AQP4. These results indicate that the majority of osmotically driven water transport in lung microvascular endothelial cells occurs by a transcellular route through AQP1 water channels. This conclusion applies to the situation in which water transport is driven by concentration gradients of small

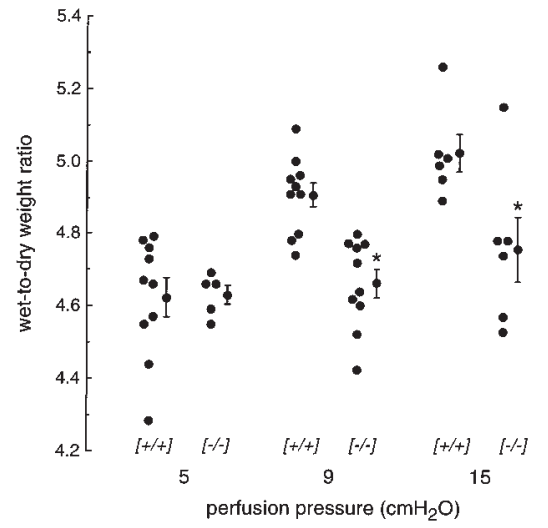

\section{Figure 4}

Effect of AQP1 deletion on hydrostatically driven lung edema by measurement of extravascular lung water. Lungs were perfused for $10 \mathrm{~min}$ at $5 \mathrm{~cm} \mathrm{H} \mathrm{H}_{2} \mathrm{O}$ and then for $5 \mathrm{~min}$ at 5,9 , or $15 \mathrm{~cm} \mathrm{H}_{2} \mathrm{O}$ as described in Methods. Individual and averaged (mean $\pm \mathrm{SE}$ ) values of extravascular lung water (expressed as corrected wet/dry ratios) are shown for indicated genotypes. ${ }^{*} P<0.001$ vs. $(+/+)$.

solutes. Because AQP1 is a water-only pore with near unity reflection coefficient for virtually all solutes (34, 35 ), water is effectively driven through the pore by sucrose and other small solutes. These same small solutes might have low reflection coefficients for driving water movement across the paracellular pathway. Oncotic forces involving large solutes such as albumin, or by hydrostatic (pressure) forces (see below), are generally believed to be important in paracellular water transport.

It has been assumed that the alveolar epithelium provides the rate-limiting barrier to water and salt movement between the airspace and capillary compartments. The results here suggest that this is not the case for osmotically driven water transport. Deletion of $100 \%$ of the AQP1 protein (in AQP1 [-/-] mice) produced a 10fold decrease in airspace-capillary osmotic water permeability. Partial deletion of AQP1 protein (in [+/-] mice) produced a slightly greater than $50 \%$ decrease in airspace-capillary osmotic water permeability. Much smaller decreases in water permeability would be expected if the alveolar epithelium were the rate-limiting barrier. Although absolute osmotic water permeability

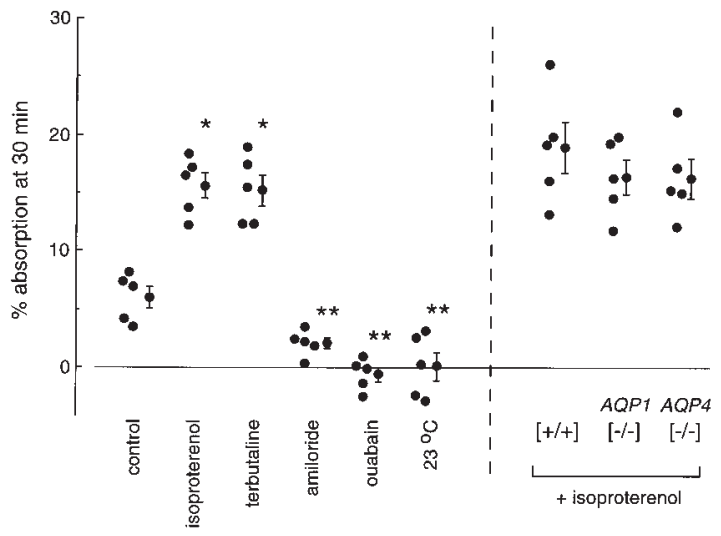

\section{Figure 5}

Effect of aquaporin deletion on active isosmolar fluid transport. Measurements were done at $37^{\circ} \mathrm{C}$ (unless otherwise indicated) using an in situ perfused lung preparation in which the airspace was instilled with an isosmolar solution containing ${ }^{125} \mathrm{I}$-albumin as a volume marker (see Methods). Percentage fluid absorption at 30 min was computed from the ratio of ${ }^{125}$ I radioactivity in fluid samples obtained at 2 and $30 \mathrm{~min}$ after instillation. (Left) Percentage absorption rates shown for individual mice along with averaged values (mean $\pm \mathrm{SE}$ ). Where indicated, the instilled and perfused solutions contained isoproterenol $(0.1 \mathrm{mM})$, terbutaline $(10 \mathrm{mM})$, amiloride $(1$ $\mathrm{mM})$, or ouabain $(0.1 \mathrm{mM})$, or the measurement was done at $23^{\circ} \mathrm{C}$ instead of $37^{\circ} \mathrm{C}$. (Right) Fluid absorption was measured at $37^{\circ} \mathrm{C}$ in the presence of $0.1 \mathrm{mM}$ isoproterenol. ${ }^{*} P<0.01$ compared with control; ${ }^{*} P<0.01 \mathrm{com}-$ pared with isoproterenol condition. 
coefficients should be viewed cautiously because of uncertainties in alveolar surface area and volume as well as heterogeneity in perfusion and alveolar geometry (23), it is useful to compare relative water permeabilities of alveolar epithelial and endothelial barriers. We reported recently (25) that $\mathrm{P}_{\mathrm{f}}$ in isolated type I alveolar epithelial cells is $\sim 0.07 \mathrm{~cm} / \mathrm{s}$ at $23^{\circ} \mathrm{C}$. Assuming that type I cells comprise the principal pathway for movement of water across the alveolar epithelium, and assuming equal apical and basolateral membrane water permeabilities, the transepithelial alveolar $\mathrm{P}_{\mathrm{f}}$ would be $\sim 0.035 \mathrm{~cm} / \mathrm{s}$. A recent measurement of microvascular water permeability gave $\mathrm{P}_{\mathrm{f}}^{\text {cap }}$ of $\sim 0.03 \mathrm{~cm} / \mathrm{s}$ at $23^{\circ} \mathrm{C}(23)$. These values are consistent with the data from AQP1 $(+/-)$ and $(-/-)$ mice, suggesting that the microvascular endothelium is an important barrier to osmotically driven airspace-capillary water transport.

The decreased airspace-capillary osmotic water permeability in AQP1 knockout mice could have a number of consequences in lung physiology. AQP1 deletion might affect hydrostatically driven lung fluid accumulation if microvascular paracellular water permeability is not much greater than transcellular water permeability. A gravimetric method was used to measure the short-term accumulation of extravascular lung water in response to increases in pulmonary artery perfusate pressure. AQP1 deletion resulted in a significant (more than fourfold) decrease in the rate of lung fluid accumulation. This conclusion was confirmed in independent measurements of extravascular lung water at a fixed time after an increase in vascular hydrostatic pressure. These findings suggest that the transcellular route may be important in hydrostatically driven fluid transport across lung microvessels. In the case of renal microvessels, where outer medullary descending vasa recta have been microdissected and perfused in vitro, osmotic water permeability driven by small solutes is very high $\left(\mathrm{P}_{\mathrm{f}} \sim 0.11 \mathrm{~cm} / \mathrm{s}\right)$ and has been attributed to transcellular water movement through AQP1 water channels (36). In response to oncotic driving forces (albumin gradients), vasa recta $\mathrm{P}_{\mathrm{f}}$ (assuming a unity albumin reflection coefficient) was $\sim 1 \mathrm{~cm} / \mathrm{s}$, suggesting a high paracellular water permeability involving wide pores (37). The data in this study suggest a different situation in lung microvessels, where AQP1-dependent transcellular water movement is most important. However, the quantitative contributions of the paracellular pathway may not be adequately assessed in these experiments because of heterogeneity in the lung vascular bed, the uncertain magnitude of vascular recruitment, and possible differences in vascular geometry in AQP1 (-/-) mice.

Another important fluid transporting function in lung is the near-isosmolar reabsorption of alveolar edema. In response to flooding of the alveolar airspaces in pulmonary edema, fluid is absorbed by an active transport process $(3,38)$. Inhibition studies and experiments in $\mathrm{ENaC}$ knockout mice have implicated a role for the $\mathrm{Na} / \mathrm{K}$ pump and the $\mathrm{ENaC}$ sodium channel in this process (5, $8)$. It is not known whether aquaporins facilitate active near-isosmolar fluid absorption in lung. From studies in rats, sheep, goats, and humans, isotonic fluid is absorbed at rates of $10 \%-20 \%$ per hour under basal conditions, increasing to $30 \%-40 \%$ per hour after stimulation by $\beta$ - agonists (3). Recently, an in situ nonperfused mouse model was developed to measure isosmolar fluid clearance (32); fluid absorption rates of $\sim 20 \%$ per hour were found, with 1.4-fold simulation by isoproterenol. In the study here, an in situ perfused mouse model was developed that gave consistent fluid absorption rates from mouse to mouse. The model was validated by showing nearly complete inhibition of fluid absorption by $\mathrm{Na}^{+}$ transport inhibitors (amiloride and ouabain) and decreased temperature, and increased fluid absorption with $\beta$-agonists (terbutaline and isoproterenol).

Despite a 10-fold reduction in airspace-capillary water permeability in AQP1 knockout mice compared with wild-type mice, the rates of isosmolar alveolar fluid absorption did not differ significantly. These results contrast with recent data in proximal tubule, where both osmotic water permeability and isomolar fluid absorption were decreased by AQP1 deletion (28). The mechanisms of near-isosmolar fluid clearance in these systems are quite different. In kidney proximal tubule, AQP1 is expressed in plasma membranes of the epithelial cells responsible for active transport. In lung, salt is actively transported from the alveolar compartment to the interstitium mainly by type II alveolar epithelial cells. Parallel movement of water probably occurs mainly through type I cells, which comprise the majority of alveolar surface area, express AQP5 (17), and are more water permeable than the type II cells (25). In the active fluid transport measurements done in this study, water moves mainly from the alveolar to interstitial compartments (39). Thus, it is not surprising that large changes in microvascular water permeability do not affect active fluid absorption across the alveolar epithelium. In addition, water transport out of the interstitium probably involves a number of processes that do not depend on transcellular water movement across the microvascular endothelium, including oncotically driven water transport into capillaries through the paracellular pathway (40), fluid clearance by pulmonary lymphatics (41), and fluid entry into the pleural space (42). We conclude that high lung microvascular water permeability is not required for active absorption of alveolar fluid, so that the principal barrier for isosmolar fluid absorption from the airspaces is the alveolar epithelium. Studies of nearisosmolar alveolar fluid absorption in AQP5 knockout mice are indicated when they become available.

\section{Acknowledgments}

We thank Jian Yang for transgenic mouse breeding and genotype analysis and Baoxue Yang for help in immunoblot analysis and immunostaining. This study was supported by National Institutes of Health grants HL-59198, HL-51854, DK-35124, HL-60288, and DK-43840, and grant R613 from the National Cystic Fibrosis Foundation.

1. Olver, R.E. 1994. Fluid secretion and absorption in the fetus. In Fluid and solute transport in the airspaces of the lung. R.M. Effros and H.K. Chang, editors. Marcel Dekker. New York, NY. 281-302.

2. Basset, G., Crone, C., and Saumon, G. 1987. Significance of active ion transport in transalveolar water absorption: a study on isolated rat lung. J. Physiol. 384:311-324.

3. Matthay, M.A., Folkesson, H., and Verkman, A.S. 1996. Salt and water transport across alveolar and distal airway epithelia in the adult lung. Am. J. Physiol. 270:L487-L503.

4. Wangensteen, O.D. 1994. Nonselective solute transport across the pul- 
monary epithelium. In Fluid and solute transport in the airspaces of the lungs. Vol. 70. R.M. Effros and H.K. Chang, editors. Marcel Dekker. New York, NY. 374-397.

5. Hummler, E., et al. 1996. Early death due to defective neonatal lung liquid clearance in $\alpha \mathrm{ENaC}$-deficient mice. Nat. Genet. 12:325-328.

6. Boucher, R.C. 1994. Human airway ion transport. Part I. Am. J. Respir. Crit. Care Med. 150:271-281.

7. Murray, C.B., et al. 1995. ClC-2: a developmentally dependent chloride channel expressed in the fetal lung and downregulated after birth. Am. J. Respir. Cell Mol. Biol. 12:597-604.

8. Ingbar, D.H., Wendt, C.H., and Crandall, E.D. 1998. Na,K-ATPase and the clearance of pulmonary edema fluid. In Pulmonary edema. M.A. Matthay and D.H. Ingbar, editors. Marcel Dekker. New York, NY. 477-500.

9. Olivera, W.G., Ridge, K.M., and Sznajder, J.I. 1995. Lung liquid clearance and $\mathrm{Na}, \mathrm{K}-\mathrm{ATP}$ ase during acute hyperoxia and recovery in rats. Am. J. Respir. Crit. Care Med. 152:1229-1234.

10. Verkman, A.S. 1998. Water transporting mechanisms in airways and lung. In Pulmonary edema. M.A. Matthay and D. Ingbar, editors. Marcel Dekker. New York, NY. 525-547.

11. Knepper, M.A. 1984. The aquaporin family of water channels. Proc. Natl. Acad. Sci. USA. 91:6255-6258.

12. Nielsen, S., et al. 1996. The aquaporin family of water channels in kidney: an update on physiology of aquaporin-2. Kidney Int. 49:1718-1723.

13. Folkesson, H.G., et al. 1994. Transcellular water transport in lung alveolar epithelium through mercury-sensitive water channels. Proc. Natl. Acad. Sci. USA. 91:4970-4974.

14. Nielsen, S., Smith, B.L., Christensen, E.I., and Agre, P. 1993. Distribution of the aquaporin CHIP in secretory and resorptive epithelia and capillary endothelia. Proc. Natl. Acad. Sci. USA. 90:7275-7279.

15. Effros, R.M., et al. 1997. Water transport and distribution of aquaporin1 in the pulmonary airspaces. J. Appl. Physiol. 83:1002-1016.

16. Frigeri, A., Gropper, M., Turck, C.W., and Verkman, A.S. 1995 Immunolocalization of the mercurial-insensitive water channel and glycerol intrinsic protein in epithelial cell plasma membranes. Proc. Natl. Acad. Sci. USA. 92:4328-4331.

17. King, L.S., Nielsen, S., and Agre, P. 1997. Aquaporins in complex tissues. I. Developmental patterns in respiratory and glandular tissues of rat. Am. J. Physiol. 273:C1541-C1548.

18. King, L.S., Nielsen, S., and Agre, P. 1996. Aquaporin-1 water channel protein in lung-ontogeny, steroid-induced expression, and distribution in rat. J. Clin. Invest. 97:2183-2191.

19. Umenishi, F., et al. 1996. Sharp increase in rat lung water channel expression in the perinatal period. Am. J. Respir. Cell Mol. Biol. 15:673-679.

20. Yasui, M., et al. 1997. Perinatal changes in expression of aquaporin-4 and other water and ion transporters in rat lung. J. Physiol. 505:3-11.

21. Carter, E.P., Umenishi, F., Matthay, M.A., and Verkman, A.S. 1997. Developmental changes in alveolar water permeability in perinatal rabbit lung. J. Clin. Invest. 100:1071-1078.

22. Carter, E.P., Matthay, M.A., Farinas, J., and Verkman, A.S. 1996. Transalveolar osmotic and diffusional water permeability in intact mouse lung measured by a novel surface fluorescence method. J. Gen. Physiol. 108:133-142.

23. Carter, E.P., Ölveczky, B.P., Matthay, M.A., and Verkman, A.S. 1998. High microvascular endothelial water permeability in mouse lung measured by a pleural surface fluorescence method. Biophys. J. 74:2121-2128.
24. Folkesson, H., Matthay, M., Frigeri, A., and Verkman, A.S. 1996. High transepithelial water permeability in microperfused distal airways: evidence for channel-mediated water transport. J. Clin. Invest. 97:664-671.

25. Dobbs, L., et al. 1998. Highly water-permeable type I alveolar epithelial cells confer high water permeability between the airspace and vasculature in rat lung. Proc. Natl. Acad. Sci. USA. 95:2991-2996.

26. Ma, T., et al. 1997. Generation and phenotype of a transgenic knock-out mouse lacking the mercurial-insensitive water channel aquaporin-4. J. Clin. Invest. 100:957-962.

27. Ma, T., et al. 1998. Severely impaired urinary concentrating ability in transgenic mice lacking aquaporin-1 water channels. J. Biol. Chem. 273:4296-4299.

28. Schnermann, J., et al. 1998. Defective proximal tubule reabsorption in transgenic aquaporin-1 null mice. Proc. Natl. Acad. Sci. USA. 95:9660-9664.

29. Chou, C.L., et al. 1998. Four-fold reduction in water permeability in inner medullary collecting duct of aquaporin-4 knockout mice. Am. J. Physiol. 274:C549-C554.

30. Gaar, K.A., Taylor, A.E., Owens, L.J., and Guyton, A.C. 1967. Pulmonary capillary pressure and filtration coefficient in the isolated perfused lung. Am. J. Physiol. 213:910-914.

31. Ehrhard, J.C., Granger, W.M., and Hofman, W.F. 1984. Filtration coefficient obtained by stepwise pressure elevation in isolated dog lung. J. Appl. Physiol. 56:862-867.

33. Shi, L.B., and Verkman, A.S. 1996. Selected cysteine point mutations confer mercurial sensitivity to the mercurial-insensitive water channel MIWC. Biochemistry. 35:538-544.

34. Van Hoek, A.N., and Verkman, A.S. 1992. Functional reconstitution of the isolated erythrocyte water channel CHIP28. J. Biol. Chem. 267:18267-18269.

35. Zeidel, M.L., Ambudkar, S.V., Smith, B.L., and Agre, P. 1992. Reconstitution of functional water channels in liposomes containing purified red cell CHIP28 protein. Biochemistry. 31:7436-7440.

36. Pallone, T.L., et al. 1997. Evidence that aquaporin-1 mediates $\mathrm{NaCl}$ induced water flux across rat descending vasa recta. Am. J. Physiol. 272:F587-F596

37. Turner, M.R., and Pallone, T.L. 1997. Hydraulic and diffusional permeabilities of isolated outer medullary descending vasa recta from the rat. Am. J. Physiol. 272:H392-H400.

38. Matalon, S., Benos, D.J., and Jackson, R.M. 1996. Biophysical and molecular properties of amiloride-inhibitable $\mathrm{Na}^{+}$channels in alveolar epithelial cells. Am. J. Physiol. 271:L1-L22.

39. Sakuma, T., et al. 1997. Beta-adrenergic agonist simulated alveolar fluid clearance in ex vivo human and rat lungs. Am. J. Respir. Crit. Care Med. 155:506-512.

40. Sakuma, T., Pittet, J.F., Jayr, C., and Matthay, M.A. 1993. Alveolar liquid and protein clearance in the absence of blood flow or ventilation in sheep. J. Appl. Physiol. 74:176-185.

41. Barthiaume, T., Staub, N.C., and Matthay, M.A. 1987. Beta-adrenergic agonists increase lung liquid clearance in anesthetized sheep. J. Clin. Invest. 779:335-343.

42. Broddus, V.C., Wiener-Kronish, J.P., and Staub, N.C. 1990. Clearance of lung edema into the pleural space of volume-loaded anesthetized sheep. J. Appl. Physiol. 68:2623-2630. 